\title{
P150 Study of Heamodynamic and Macrocirculation Indices Between Uncontrolled Naïve Hypertensives and Well Controlled Diabetic Patients
}

Areti Triantafyllou, Nick Koletsos, Konstantina Dipla, Ioanna Zografou, Xristos Avgerinos, Stauros Papadopoulos, Alexandros-Savvas Zafeiridis, Stella Kritikou, Eugenia Gkaliagkousi, Andreas Zafeiridis, Stella Douma

Aristotle University of Thessaloniki, Greece

\section{ABSTRACT}

Introduction: Both Type II Diabetes mellitus (DM) and hypertension have been associated with an increased risk for cardiovascular disease and linked to impairments in micro- and macrocirculation. However, previous studies examining microand macrocirculation in DM mainly include patients with comorbid hypertension. Therefore, the aim of the study was to compare hemodynamic and vascular indices in DM and non-DM patients, independently of their hypertension status.

Materials and Methods: Fifty-seven DM patients and 79 non-DM participants were enrolled. Participants underwent physical examination, ambulatory BP monitoring and estimation of pulse wave velocity (PWV) and intima-media thickness (IMT). Resting hemodynamic parameters were assessed by impedance cardiography. Participants also performed a 3-min-submaximal isometric handgrip ( $30 \% \mathrm{MVC}$ ) with continuous beat-by-beat BP/HR assessment (Finapress). The double product (DP = systolic blood pressure heart rate) during HG was calculated, as an index of myocardial stress.

Results: No differences were observed in age, BMI, and resting BP among groups. Patients with DM had significantly higher PWV and IMT $(p<0.01)$ but lower velocity (VI) and acceleration index (ACI) compared to non-DM individuals $(p<0.05)$. Hypertensives had significantly higher myocardial stress during exercise compared to normotensives, independently of the presence of diabetes.

Conclusion: Despite similar blood pressure levels in DM and non-DM groups, the DM patients had higher PWV and IMT than non-DM participants. There were no differences between patients with isolated adequately controlled DM or isolated hypertension in macrocirculation indices, suggesting a possible equal impact of the above diseases on the macrovascular network.

\section{REFERENCES}

[1] Karavelioglu Y, Karapinar H, Gul İ, Kucukdurmaz Z, Yilmaz A, Akpek M, et al. Blood pressure response to exercise is exaggerated in normotensive diabetic patients. Blood Press 2013;22:21-6.

[2] Fang ZY, Prins JB, Marwick TH. Diabetic cardiomyopathy: evidence, mechanisms, and therapeutic implications. Endocr Rev 2004;25:543-67.

(C) 2019 Association for Research into Arterial Structure and Physiology. Publishing services by Atlantis Press International B.V. This is an open access article distributed under the CC BY-NC 4.0 license (http://creativecommons.org/licenses/by-nc/4.0/). 